\title{
The Time-dependent Hartree-Fock Equations with Coulomb Two-Body Interaction
}

\section{J. M. Chadam*}

Centre de Physique Théorique, C.N.R.S.,

F-13274 Marseille Cedex 2, France

\begin{abstract}
The existence and uniqueness of global solutions to the Cauchy problem is proved in the space of "smooth" density matrices for the timedependent Hartree-Fock equations describing the motion of finite Fermi systems interacting via a Coulomb two-body potential.
\end{abstract}

\section{Introduction}

In this note, we indicate how to generalize the recent results of Bove, Da Prato, and Fano [1] concerning the time-dependent Hartree-Fock equations with bounded two-body interaction to include the Coulomb two-body interaction. (See this work and the references therein for a discussion of the origin of the problem.) Specifically we consider the existence of global solutions to the Cauchy problem for the equations

$$
i d K / d t=\left[\frac{1}{2} \Delta-U, K\right]_{-},
$$

where $K=K(t)$ is a density matrix [i.e. a non-negative trace class operator on $\left.L^{2}\left(R^{3}\right)\right]$ and $U$ is the self-consistent potential $U_{D}-U_{\mathrm{EX}}$ defined by

$$
\left(U_{D} f\right)(x)=\left(\int|x-y|^{-1} k(y, y ; t) d y\right) f(x)
$$

and

$$
\left(U_{\mathrm{EX}} f\right)(x)=-\int|x-y|^{-1} k(x, y ; t) f(y) d y
$$

when $K(t)$ is represented as the integral operator $(K(t) f)(x)=\int k(x, y ; t) f(y) d y$. The idea of the argument is to extend to this situation our results [2] for $N$ electron systems governed by the Hartree-Fock equations

$$
i \partial \varphi_{j} / \partial t=\frac{1}{2} \Delta \varphi_{j}-U_{\mathrm{op}} \varphi_{j}
$$

* On leave of absence from Mathematics Department, Indiana University, Bloomington, Indiana 47401, USA. 
where

$$
\begin{aligned}
U_{\mathrm{op}} \varphi_{j}(x, t)= & \sum_{l=1}^{N}\left(\varphi_{j}(x, t) \int|x-y|^{-1}\left|\varphi_{l}(y, t)\right|^{2} d y\right. \\
& \left.-\varphi_{l}(x, t) \int|x-y|^{-1} \bar{\varphi}_{l}(y, t) \varphi_{j}(x, t) d y\right) .
\end{aligned}
$$

The connection between the problems (1.1)-(1.3) and (1.4), (1.5) is the following: Suppose $\varphi_{j}(x, t)$ is the unique global solution of the latter Cauchy problem with data $\varphi_{j}(x, 0)=\sqrt{\lambda_{j}} \varphi_{j}^{0}(x)$, then $k_{N}(x, y ; t)=\sum_{j=1}^{N} \varphi_{j}(x, t) \bar{\varphi}_{j}(y, t)$ is the kernel of a solution of the original Cauchy problem with data $K_{N}^{0}$ having kernel $\sum_{j=1}^{N} \lambda_{j} \varphi_{j}^{0}(x) \bar{\varphi}_{j}^{0}(y)$. In this framework, the results of [2] can be interpreted as a solution of the problem (1.1)-(1.3) within a certain class of finite-rank operators. Section 2 of this paper consists of making precise this idea as well as that of taking the limit $N \rightarrow \infty$. The key ingredient in the limiting procedure will be the a priori estimates developed in Lemma 3.4 of [2].

\section{The Results}

It is well-known that in order to handle the Coulomb potential one must ultimately introduce derivatives (see, for example, the calculations of Lemma 2.3 of [2] which are typical). For this reason the solution space is taken to be the following Banach space of "smooth" operators.

Definition 2.1. Let $A^{2}$ denote the self-adjoint realization of $I-\Delta$ on $L^{2}\left(\mathbb{R}^{3}\right)$. Suppose $\mathscr{L}\left(L^{2}\left(\mathbb{R}^{3}\right)\right)$ is the set of all bounded operators on $L^{2}\left(\mathbb{R}^{3}\right)$ and $\mathscr{L}^{1}\left(L^{2}\left(\mathbb{R}^{3}\right)\right)$ is the set of trace class operators on $L^{2}\left(\mathbb{R}^{3}\right)$. Define $S=\left\{K ; K \in \mathscr{L}\left(L^{2}\left(\mathbb{R}^{3}\right)\right)\right.$ and $\left.A|K| A \in \mathscr{L}^{1}\left(L^{2}\left(\mathbb{R}^{3}\right)\right)\right\}$ with the norm in $S$ taken to be $\|K\|_{1,1}=\operatorname{tr}(A|K| A)=$ $\|A|K| A\|_{1}$.

In what follows we use $\|\cdot\|$ to denote the norm in $L^{2}\left(\mathbb{R}^{3}\right)$ and $\mathscr{L}\left(L^{2}\left(\mathbb{R}^{3}\right)\right)$, $\|\cdot\|_{1}$ the norm in $\mathscr{L}^{1}\left(L^{2}\left(\mathbb{R}^{3}\right)\right)$ and $\|\cdot\|_{1,1}$ for the norm in $S$ in as much as it corresponds to the Sobolev space $H^{1}\left(\mathbb{R}^{3}\right)$ of scalar functions. Indeed for physical reasons we are only interested in the cone of positive operators in $S$, denoted by $S^{+}$and called smooth density matrices. Before beginning the main discussion, we summarize some ideas about trace class operators (cf. [3]) which will be useful in the later calculations. Since $|K| \geqq 0$, then $A|K| A \geqq 0$ so that $\|K\|_{1,1}=\operatorname{tr}(A|K| A)=$ $\|A|K| A\|_{1}$. But $A^{-1}$ is bounded on $L^{2}\left(\mathbb{R}^{3}\right)$ so that $|K|=A^{-1} A|K| A A^{-1}$ and hence $K \in \mathscr{L}^{1}\left(L^{2}\left(\mathbb{R}^{3}\right)\right)$. Thus $K$ can be written as an integral operator $(K f)(x)=$ $\int k(x, y) f(y) d y$, with kernel $k(x, y) \in L^{2}\left(\mathbb{R}^{3}\right) \times L^{2}\left(\mathbb{R}^{3}\right)$ and $|k|(x, x) \in L^{1}\left(\mathbb{R}^{3}\right)$. Moreover $k(x, y)=\sum_{j=1}^{\infty} \lambda_{j} \varphi_{j}(x) \bar{\varphi}_{j}(y)$ and the kernel associated with $|K|,|k|(x, y)=$ $\sum_{j=1}^{\infty}\left|\lambda_{j}\right| \varphi_{j}(x) \bar{\varphi}_{j}(y)$ [where $\left\{\left|\lambda_{j}\right|, \varphi_{j}\right\}$ is a spectral set for $|K|$ and the convergence is in $L^{2}\left(\mathbb{R}^{3}\right) \times L^{2}\left(\mathbb{R}^{3}\right)$ with $\left.\sum\left|\lambda_{j}\right|=\sum\left|\lambda_{j}\right|\left\|\varphi_{j}\right\|^{2}=\int|k|(x, x) d x<\infty\right]$. Finally, because $|K| \varphi_{j}=\left|\lambda_{j}\right| \varphi_{j}, \quad A \varphi_{j}=\left|\lambda_{j}\right|^{-1} A|K| \varphi_{j}=\left|\lambda_{j}\right|^{-1} A|K| A A^{-1} \varphi_{j}$. Thus $\left\|A \varphi_{j}\right\| \leqq$ $\left|\lambda_{j}\right|^{-1}\|A|K| A\|\left\|A^{-1} \varphi_{j}\right\| \leqq\left|\lambda_{j}\right|^{-1}\|A|K| A\|_{1}\left\|\varphi_{j}\right\|=\left|\lambda_{j}\right|^{-1}\|K\|_{1,1}$, so that $\varphi_{j} \in D(A)$. Thus the kernel of $A|K| A$ is $\sum\left|\lambda_{j}\right| A \varphi_{j}(x) \overline{A \varphi_{j}}(y)$ and $\|K\|_{1,1}=\sum\left|\lambda_{j}\right|\left\|A \varphi_{j}\right\|^{2}$. 
Definition 2.2. $K(t)$ is a solution of the Cauchy problem (1.1)-(1.3) over the interval $(0, T)$ if the map $t \rightarrow K(t):(0, T) \rightarrow S$ is continuous and $K(t)$ satisfies the integrated form of Eq. (1.1),

$$
\begin{aligned}
K(t)= & e^{-i \Delta / 2 t} K(0) e^{i \Delta / 2 t} \\
& +i \int_{0}^{t} e^{-i \Delta / 2(t-s)}[U, K](s) e^{i \Delta / 2(t-s)} d s,
\end{aligned}
$$

the last integral being interpreted in the strong Riemann sense in $S$.

Proposition 2.3. The Eq. (2.1) has a unique local solution in S.

Proof. Segal's generalization of the Picard-Lipschitz theory to infinite dimensional spaces [4, p. 343, Theorem 1] can be applied directly. First the free propagator is a contraction group since $\left\|e^{-i \Delta / 2 t} K e^{i \Delta / 2 t}\right\|_{1,1}=\left\|A\left|e^{-i \Delta / 2 t} K e^{i \Delta / 2 t}\right| A\right\|_{1}=$ $\left\|A e^{-i \Delta / 2 t}|K| e^{i \Delta / 2 t} A\right\|=\left\|e^{-i \Delta / 2 t} A|K| A e^{i \Delta / 2 t}\right\|_{1}$ by the spectral theorem and the last equals $\|A|K| A\|_{1}=\|K\|_{1,1}$ by [1, p. 186, Proposition 3.4]. The local Lipschitz nature of the non-linearity follows essentially as usual. By straightforward algebra

$$
[U(T), T]-[U(S), S]=[U(T), T-S]-[U(T-S), S],
$$

so that it is enough to show that $\|U(K) L\|_{1,1}$ and $\|L U(K)\|_{1,1} \leqq C\|K\|_{1,1}\|L\|_{1,1}$. Now $\|U(K) L\|_{1,1}=\operatorname{tr}(A|U(K) L| A)=\operatorname{tr}\left(|U(K) L| A^{2}\right) \leqq\|U(K)\|\|K\|_{1}$ extracting the partial isometries in the polar decomposition of $U(K)$ and $U(K) L$ from the left. Similarly $\|L U(K)\|_{1,1}=\operatorname{tr}\left(A^{2}|L U(K)|\right) \leqq\|L\|_{1,1}\|U(K)\|$. Thus one must show that $\left\|U_{D}(K)\right\|$ and $\left\|U_{\mathrm{EX}}(K)\right\| \leqq$ const $\|K\|_{1,1}$. This follows directly from the Sobolev type estimates in [2, Lemma 2.3].

Suppose $K$ has a kernel $\sum \lambda_{j} \psi_{j}(x) \bar{\varphi}_{j}(y)$ where $\left\{\left|\lambda_{j}\right|, \varphi_{j}\right\}$ is a spectral set for $|K|$ and $\left\{\psi_{j}\right\}$ is an orthonormal basis in $L^{2}\left(\mathbb{R}^{3}\right) . U_{D}(K)$ is multiplication by $\sum \lambda_{j} \int|x-y|^{-1} \psi_{j}(y) \bar{\varphi}_{j}(y) d y$ and so

$$
\begin{aligned}
\left\|U_{D}(K)\right\| & \leqq \sum\left|\lambda_{j}\right| \sup _{x} \int|x-y|^{-1}\left|\psi_{j}(y)\right|\left|\varphi_{j}(y)\right| d y \\
& \leqq \sum\left|\lambda_{j}\right|\left\|\psi_{j}\right\|\left\|\int|x-y|^{-1}\left|\varphi_{j}(y)\right| d y\right\| \\
& \leqq C \sum\left|\lambda_{j}\right|\left\|\nabla \varphi_{j}\right\| \\
& \leqq C \sum\left|\lambda_{j}\right|^{\frac{1}{2}}\left(\left|\lambda_{j}\right|\left\|\nabla \varphi_{j}\right\|^{2}\right)^{\frac{1}{2}} \\
& \leqq \frac{1}{2} C \sum\left|\lambda_{j}\right|\left(1+\left\|\nabla \varphi_{j}\right\|^{2}\right)=\frac{1}{2} C\|K\|_{1,1} .
\end{aligned}
$$

For the exchange term,

$$
\begin{aligned}
\left\|U_{\mathrm{EX}}(K) f\right\| & =\left\|\int \sum \lambda_{j}|x-y|^{-1} \psi_{j}(x) \bar{\varphi}_{j}(y) f(y) d y\right\| \\
& \leqq \sum\left|\lambda_{j}\right|\left\|\psi_{j}\right\| \sup _{x} \int|x-y|^{-1}\left|\varphi_{j}(y)\right||f(y)| d y \\
& \leqq C \sum\left|\lambda_{j}\right|\left\|\nabla \varphi_{j}\right\|\|f\| \\
& \leqq \frac{1}{2} C\|K\|_{1,1}\|f\| .
\end{aligned}
$$

The fact that if $K$ at $t=0$ is positive it remains positive in the interval of existence is proved in [1].

In proving that this solution can be extended to all of $(0, \infty)$ we shall make use of the following representation of finite rank solutions in $S^{+}$. 
Proposition 2.4. Suppose the initial data $K^{0}$ is a finite rank operator in $\mathrm{S}^{+}$; i.e. $K^{0}=\sum_{j=1}^{N} \lambda_{j} \varphi_{j}^{0}(x) \overline{\varphi_{j}^{0}}(y)$ where $\left\{\lambda_{j} \geqq 0, \varphi_{j}^{0}\right\}_{j=1}^{N}$ is a spectral set in $L^{2}\left(\mathbb{R}^{3}\right)$ with $\varphi_{j}^{0} \in D(A) \cong H^{1}$ for all $j=1, \ldots, N$. Denote by $\varphi_{j}(x, t), j=1,2, \ldots N$ the unique (global) solution of the (integral form of) Eq. (1.4) in $H^{1}\left(\mathbb{R}^{3}\right)$ with initial data $\sqrt{\lambda_{j}} \varphi_{j}^{0}(x)$ as given in [2]. Then

$$
K(t)=\sum_{j=1}^{N} \varphi_{j}(x, t) \overline{\varphi_{j}}(y, t)=\sum_{j=1}^{N} \lambda_{j}\left(\varphi_{j}(x, t) / \sqrt{\lambda_{j}}\right)\left(\overline{\varphi_{j}}(y, t) / \sqrt{\lambda_{j}}\right)
$$

is the unique global solution in $S^{+}$of (the integral form of ) problem (1.1)-(1.3) with initial data $\mathrm{K}^{0}$.

Proof. The idea of the proof can be seen most easily from the viewpoint of the differential equations and the proof for the integral equations involves only simple but non-essential algebraic considerations. From (1.4)

$$
\begin{aligned}
i \partial \varphi_{j}(x, t) / \partial t \overline{\varphi_{j}}(y, t)= & \frac{1}{2} \Delta \varphi_{j}(x, t) \overline{\varphi_{j}}(y, t)-\sum_{l=1}^{N} \varphi_{j}(x, t) \overline{\varphi_{j}}(y, t) \int|x-z|^{-1}\left|\varphi_{l}(z, t)\right|^{2} d z \\
& +\sum_{l=1}^{N} \varphi_{l}(x, t) \overline{\varphi_{j}}(y, t) \int|x-z|^{-1} \overline{\varphi_{l}}(z, t) \varphi_{j}(z, t) d z
\end{aligned}
$$

Taking conjugates, exchanging $x$ and $y$, adding the new equation to the above and summing over $j$ from $l$ to $N$ one obtains

$$
\begin{aligned}
i \partial / \partial t & \sum_{j=1}^{N} \varphi_{j}(x, t) \overline{\varphi_{j}}(y, t)=\frac{1}{2}\left(\sum_{j} \Delta \varphi_{j}(x, t) \varphi_{j}(y, t)-\sum_{j} \varphi_{j}(x, t) \overline{\Delta \varphi_{j}}(y, t)\right) \\
& -\int\left(|x-z|^{-1} \sum_{j} \varphi_{j}(x, t) \overline{\varphi_{j}}(y, t) \sum_{l} \varphi_{l}(z, t) \overline{\varphi_{l}}(z, t)\right. \\
& \left.-|y-z|^{-1} \sum_{j} \varphi_{j}(x, t) \overline{\varphi_{j}}(y, t) \sum_{l} \varphi_{l}(z, t) \overline{\varphi_{l}}(z, t)\right) d z, \\
& +\int\left(|x-z|^{-1} \sum_{j} \varphi_{j}(z, t) \overline{\varphi_{j}}(y, t) \sum_{l} \varphi_{l}(x, t) \overline{\varphi_{l}}(z, t)\right. \\
& \left.-|y-z|^{-1} \sum_{j} \varphi_{j}(x, t) \overline{\varphi_{j}}(z, t) \sum_{l} \varphi_{l}(z, t) \overline{\varphi_{l}}(y, t)\right) d z,
\end{aligned}
$$

which is just Eq. (1.1) written for the kernel $\sum_{j} \varphi_{j}(x, t) \overline{\varphi_{j}}(y, t)$. From [2, Lemmas 3.1 and 3.4] $\left\|\varphi_{j}(t) / \sqrt{\lambda_{j}}\right\|=\left\|\varphi_{j}^{0}\right\|=1$ and $\varphi_{j}(t) \in D(A)$ for all $t$. One can also show in the same manner that since the $\left\{\varphi_{j}^{0}\right\}$ are orthogonal, the $\left\{\varphi_{j}(t)\right\}$ are orthogonal for each $t$. Thus $K(t)=\sum \lambda_{j}\left(\varphi_{j}(x, t) / \sqrt{\lambda_{j}}\right)\left(\bar{\varphi}_{j}(y, t) / \sqrt{\lambda_{j}}\right)$ is the unique global solution of (1.1) in $S^{+}$with the given Cauchy data.

Theorem 2.5. The Cauchy problem for (the integral version of) the Eq. (1.1) has a unique global solution in $S^{+}$.

Proof. Suppose the Cauchy data at $t=0$ is $K^{0}=\sum_{j=1}^{\infty} \lambda_{j} \varphi_{j}^{0}(x) \overline{\varphi_{j}^{0}}(y)$ where, since $K^{0} \in S^{+},\left\{\lambda_{j} \geqq 0, \varphi_{j}^{0}\right\}$ is a spectral set and $\left\|K^{0}\right\|_{1,1}=\sum_{j=1}^{\infty} \lambda_{j}\left(1+\left\|\nabla \varphi_{j}^{0}\right\|^{2}\right)<\infty$. 
Then $\left\{K_{N}^{0}=\sum_{j=1}^{N} \lambda_{j} \varphi_{j}^{0}(x) \overline{\varphi_{j}^{0}}(y)\right\}_{N=1}^{\infty}$ is a sequence of finite rank operators approximating $K^{0}$ in $S$. From the above $K_{N}(t)=\sum_{j=1}^{N} \lambda_{j}\left(\varphi_{j}(x, t) / \sqrt{\lambda_{j}}\right)\left(\overline{\varphi_{j}}(y, t) / \sqrt{\lambda_{j}}\right)$ is the unique global solution of Eq. (1.1) with data $K_{N}^{0}$ at $t=0$. The theorem will be proved if we can show that for each $t \in(0, \infty), K_{N}(t)$ converges in $S$ (indeed, we will show that the convergence is uniform in $t$ ) and that the limiting operator function of $t$ is a solution of (1.1).

To this end consider, for any $t \in(0, \infty)$,

$$
\begin{aligned}
\left\|K_{N}(t)-K_{M}(t)\right\|_{1,1} & =\sum_{j=M}^{N} \lambda_{j}\left(\left\|\varphi_{j}(t) / \sqrt{\lambda_{j}}\right\|^{2}+\left\|\nabla \varphi_{j}(t) / \sqrt{\lambda_{j}}\right\|^{2}\right) \\
& =\sum_{j=M}^{N} \lambda_{j}\left(1+\lambda_{j}^{-1}\left\|\nabla \varphi_{j}(t)\right\|^{2}\right) \\
& =\sum_{j=M}^{N} \lambda_{j}+\sum_{j=M}^{N}\left\|\nabla \varphi_{j}(t)\right\|^{2},
\end{aligned}
$$

where we have used the estimate $\left[2\right.$, Lemma 3.1] $\left\|\varphi_{j}(t) / \sqrt{\lambda_{j}}\right\|=\left\|\varphi_{j}^{0}\right\|=1$. From [2, Lemma 3.4], since $\varphi_{j}(t)$ is a solution of (1.4),

$$
\sum_{j=1}^{N}\left\|\nabla \varphi_{j}(t)\right\|^{2}+\sum_{j=1}^{N} \sum_{l=1}^{N} I_{j, \bar{l}}(t)=\sum_{j=1}^{N}\left\|\nabla \varphi_{j}(0)\right\|^{2}+\sum_{j=1}^{N} \sum_{l=1}^{N} I_{j, l}(0),
$$

where $I_{j, l}(t)=\int v_{l}(x, t)\left|\varphi_{j}(x, t)\right|^{2}-1 / 4 \pi\left|\nabla v_{j l}(x, t)\right|^{2} d x$, with $v_{l}(x, t)$ and $v_{j, l}(x, t)$ being respectively the first and second integral in Eq. (1.5). Now $I_{j, l}(t) \geqq 0$ (cf. [2], from Eq. (3.7) on) for each $j, l$, so that

$$
\begin{aligned}
\sum_{j=M}^{N}\left\|\nabla \varphi_{j}(t)\right\|^{2} & \leqq \sum_{j=N}^{N}\left\|\nabla \varphi_{j}(0)\right\|^{2}+\sum_{j=1}^{N} \sum_{l=1}^{N} I_{j, l}(0)-\sum_{j=1}^{M} \sum_{l=1}^{M} I_{j, l}(0) \\
& \leqq \sum_{j=M}^{N} \lambda_{j}\left\|\nabla \varphi_{j}^{0}\right\|^{2}+\sum_{j=M}^{N} \sum_{l=1}^{M} I_{j, l}(0)+\sum_{j=1}^{N} \sum_{l=M}^{N} I_{j, l}(0) .
\end{aligned}
$$

But $I_{j, l}(0)=\int\left\{\left(\int|x-y|^{-1}\left|\varphi_{l}(x, 0)\right|^{2} d y\right)\left|\varphi_{j}(x, 0)\right|^{2}-1 / 4 \pi\left|\nabla \int\right| x-\left.y\right|^{-1} \overline{\varphi_{l}}(y, 0) \varphi_{j}(y, 0)\right.$. $\left.\left.d y\right|^{2}\right\} d x$, so that

$$
\begin{aligned}
I_{j, l}(0) & \leqq \lambda_{j} \lambda_{l}\left(\sup _{x} \int|x-y|^{-1}\left|\varphi_{l}^{0}(y)\right|^{2} d y\left\|\varphi_{j}^{0}\right\|^{2}+1 / 4 \pi\left\|\int|x-y|^{-2}\left|\varphi_{l}^{0}(y)\right|\left|\varphi_{j}^{0}(y)\right| d y\right\|^{2}\right) \\
& \leqq \lambda_{j} \lambda_{l}\left(2\left\|\varphi_{l}^{0}\right\|\left\|\varphi_{j}^{0}\right\|^{2}\left\|\nabla \varphi_{l}^{0}\right\|+c / 4 \pi\left\|\varphi_{l}^{0} \varphi_{j}^{0}\right\|_{6 / 5}^{2}\right) \\
& \leqq C \lambda_{j} \lambda_{l}\left(\left\|\varphi_{l}^{0}\right\|\left\|\nabla \varphi_{l}^{0}\right\|\left\|\varphi_{j}^{0}\right\|^{2}+\left\|\varphi_{l}^{0}\right\|_{12 / 5}^{2}\left\|\varphi_{j}^{0}\right\|_{12 / 5}^{2}\right) \\
& \leqq C \lambda_{j} \lambda_{l}\left(\left\|\varphi_{l}^{0}\right\|\left\|\nabla \varphi_{l}^{0}\right\|\left\|\varphi_{j}^{0}\right\|^{2}+\left\|\nabla \varphi_{l}^{0}\right\|^{1 / 2}\left\|\varphi_{l}^{0}\right\|^{3 / 2}\left\|\nabla \varphi_{j}^{0}\right\|^{1 / 2}\left\|\varphi_{j}^{0}\right\|^{3 / 2}\right) \\
& \leqq C \lambda_{j} \lambda_{l}\left(\left\|\varphi_{l}^{0}\right\|^{2}+\left\|\nabla \varphi_{l}^{0}\right\|^{2}\right)\left(\left\|\varphi_{j}^{0}\right\|^{2}+\left\|\nabla \varphi_{j}^{0}\right\|^{2}\right),
\end{aligned}
$$

where we have used Sobolev inequalities as they appear in $[5$, p. 220] and $[6$, p. 27 , Theorem 10.1] in a manner like [2] and the inequality $a^{1 / l} b^{1 / m} \leqq a / l+b / m$ if $1 / l+1 / m=1$. The constant $C$ changes from line to line. Thus

$$
\left\|K_{N}(t)-K_{M}(t)\right\|_{1,1} \leqq C\left(1+\left\|K^{0}\right\|_{1,1}\right)\left\|K_{N}^{0}-K_{M}^{0}\right\|_{1,1}
$$


showing that $\left\{K_{N}(t)\right\}$ is Cauchy in $S$ uniformly in $t \in(0, \infty)$ since $K_{N}^{0} \rightarrow K^{0}$ in $S$. As a result $\left\{K_{N}(t)\right\}$ converges in $S$ uniformly in $t \in(0, \infty)$ to an operator which is continuous in $t \in(0, \infty)$ [since for each $N, K_{N}(t)$ is continuous in $S$ ] and positive. The uniformity in $t$ of the convergence, the continuity of the non-linearity in $S$ and the invariance of the $\|\cdot\|_{1,1}$-norm under the free motion (Proposition 2.3) guarantee that the limiting operator is a solution of Eq. (2.1) in $S$. Thus it is the (necessarily unique) global solution of the Cauchy problem in $S^{+}$of Eq. (1.1)-(1.3).

In conclusion we remark that other two body potentials (e.g. Yukawa) along with the inclusion of a central potential can be treated in this manner by suitably adjusting the "Sobolev spaces" $S_{n, p}=\left\{K \in \mathscr{L}\left(L^{2}\left(\mathbb{R}^{3}\right)\right),\|K\|_{n, p}^{p}=\operatorname{tr}\left(A^{n}|K|^{p} A^{n}\right)<\infty\right\}$ in a manner suggested by the classical (i.e. scalar or vector) theory of partial differential equations.

Acknowledgements. I like to thank the faculties of many institutions for the kind hospitality extended to me during the various stages of the preparation of this work, especially Professors J. M. Combes at the Centre Universitaire de Toulon, R. Raczka at the Institute of Nuclear Research in Warsaw, R. Stora at the Centre de Physique Théorique, CNRS Marseille. I am very much indebted to Professors G. Da Prato and A. Bove for the opportunity to discuss this work with them at the Mathematics Institute in Rome. I am grateful for financial support from the N.S.F. (USA), D.G.R.S.T. (France), C.N.R.S. (France).

\section{References}

1. Bove,A., Da Prato,G., Fano,G.: An existence proof for the Hartree-Fock time-dependent problem with bounded two-body interaction. Commun. math. Phys. 37, 183 (1974)

2. Chadam, J., Glassey, R.: Global existence of solutions to the Cauchy problem for time dependent Hartree equations. J. Math. Phys. 16, 1122 (1975)

3. Schatten, R.: Norm ideals of completely continuous operators. Berlin-Göttingen-Heidelberg: Springer 1960

4. Segal, I.: Nonlinear semigroups. Ann. Math. 78, 339 (1963)

5. Bers, L., John, F., Schecter, M.: Partial differential equations. New York: Interscience 1964

6. Friedman, A.: Partial differential equations. New York, etc.: Holt, Rinehart, Winston 1969

Communicated by W. Hunziker

Received August 1, 1975 